\title{
Solution-Based Growth and Structural Characterization of Homo- and Hetero-branched Semiconductor Nanowires
}

\author{
Angang Dong, Rui Tang, and William E. Buhro \\ Department of Chemistry and Center for Materials Innovation, Washington University, \\ St. Louis, Missouri 63130-4899
}

\section{Table of Contents}

Figure S1. Representative TEM image of the ZnSe NW-Bi composite obtained by co-precipitation method after annealing . .2

Figure S2. Branch-diameter distribution histogram of the homobranched ZnSe

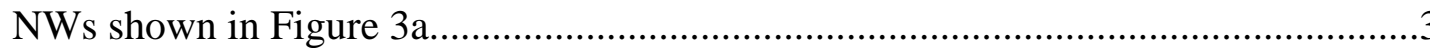

Figure S3. EDS spectra collected from the selected spots on a single heterobranched CdSe-ZnSe NW shown in Figure 9a ................................................4

Figure S4. HRTEM image of a heterobranched CdSe-ZnSe NW with stacking faults

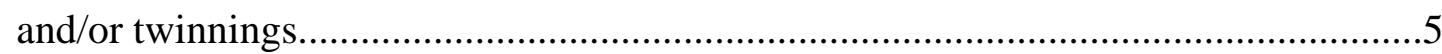

Figure S5. Cartoon illustration of the orientation and growth directions of the heterobranched CdSe-ZnSe NW shown in Figure 11 .............................................6 Figure S6. HRTEM image of another heterobranched CdSe-ZnSe NW with the backbone having a hexagonal crystal structure 


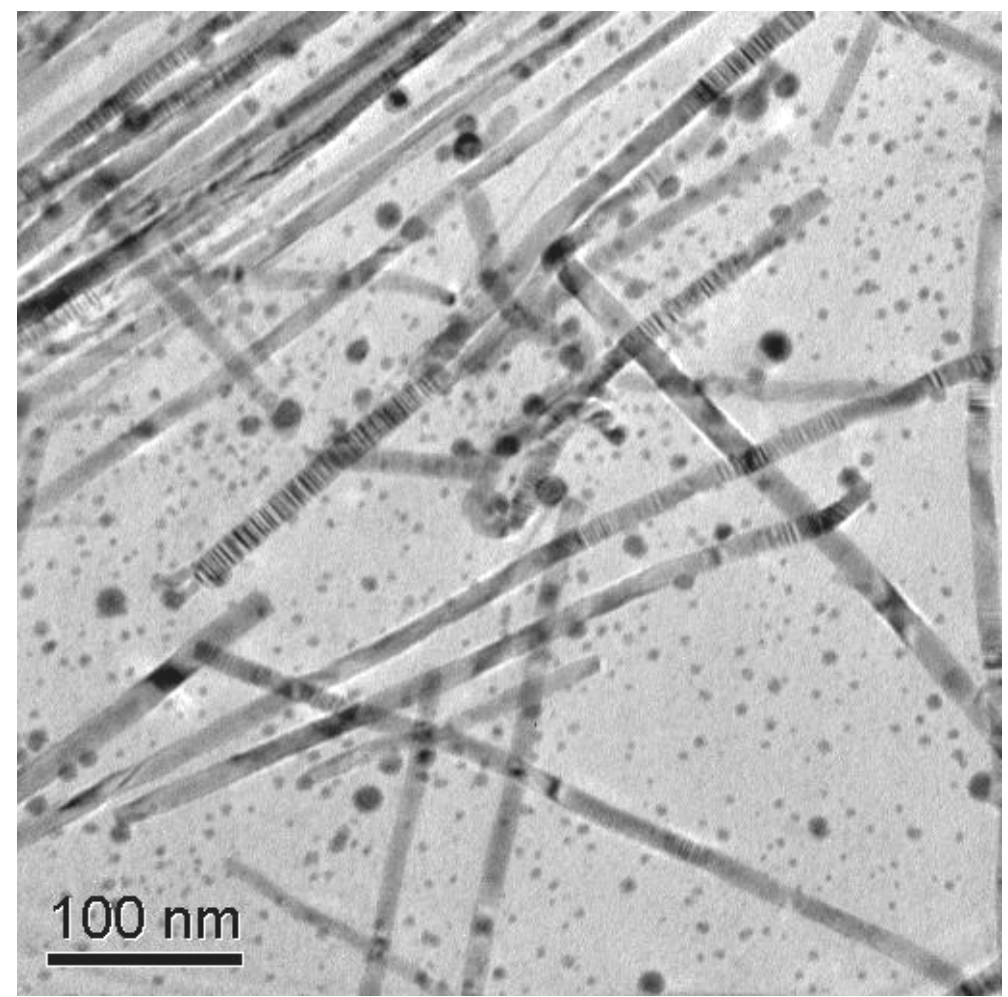

Figure S1. Representative TEM image of the ZnSe NW-Bi composite obtained by the co-precipitation method followed by annealing, showing that most of the Bi seeds ( $\sim 7 \mathrm{~nm}$ in diameter) were not attached to the NW surface due to the weak interaction between the Bi seeds and NW backbones. Note that some Bi nanoparticles became larger, presumably due to aggregation and/or Ostwald ripening during the high-temperature annealing process. 


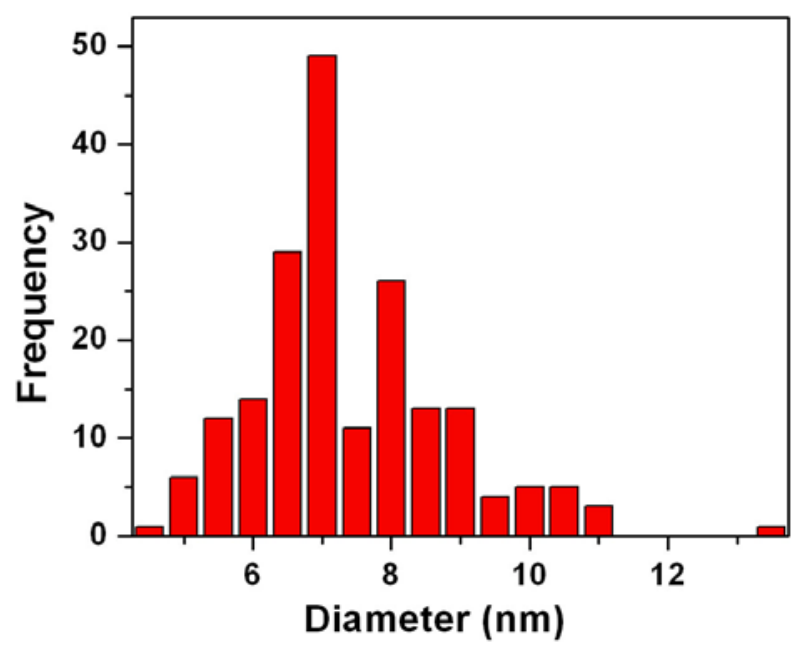

Figure S2. Branch-diameter distribution histogram of the homobranched ZnSe NWs shown in Figure 3a. Mean branch diameter $=7.2 \pm 1.4 \mathrm{~nm}$. 

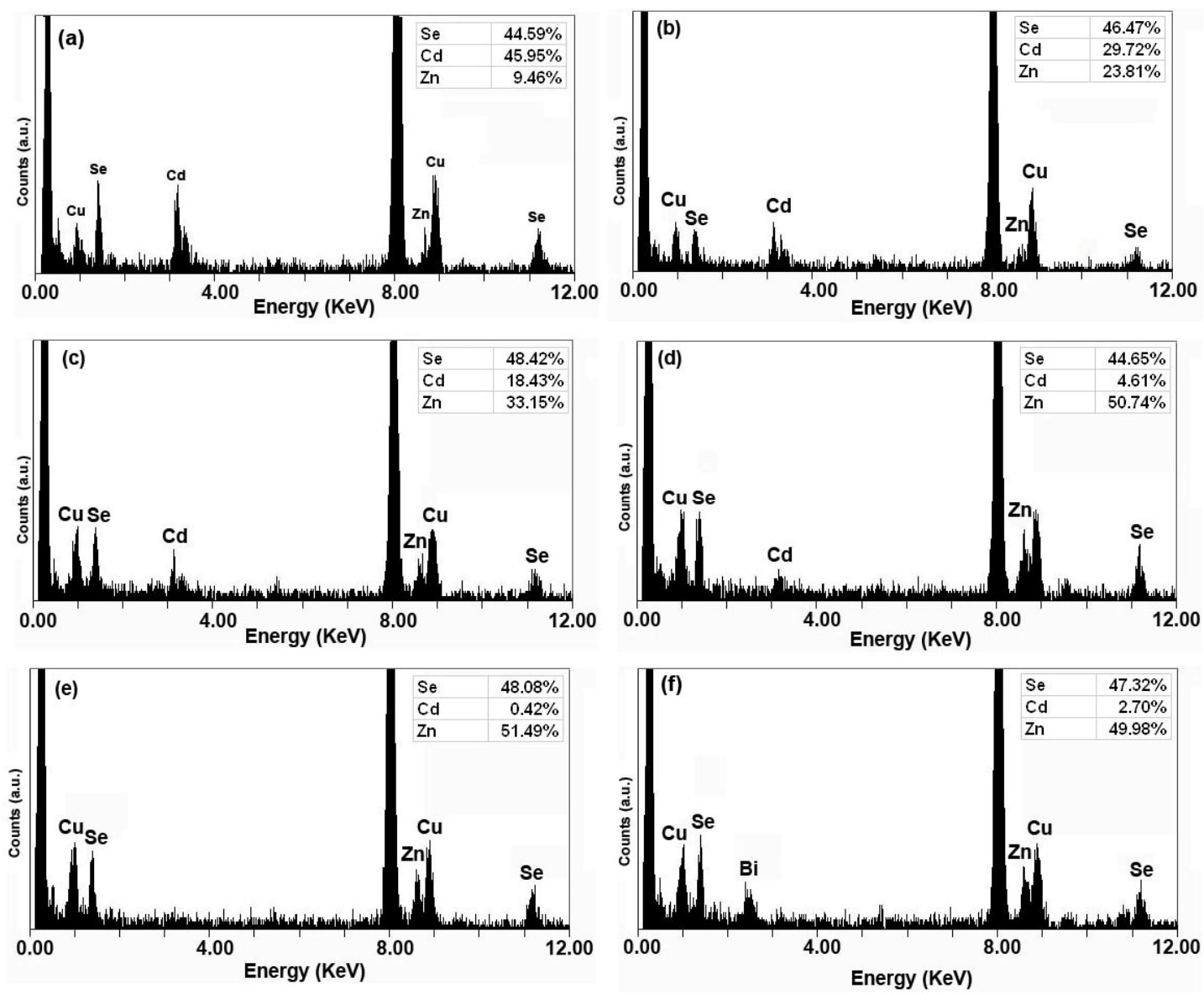

Figure S3. (a)-(f) EDS spectra collected from selected spots on the single heterobranched CdSe-ZnSe NW shown in Figure 9a. These data were used to construct the EDS line-scan profiles shown in Figure 9b. 


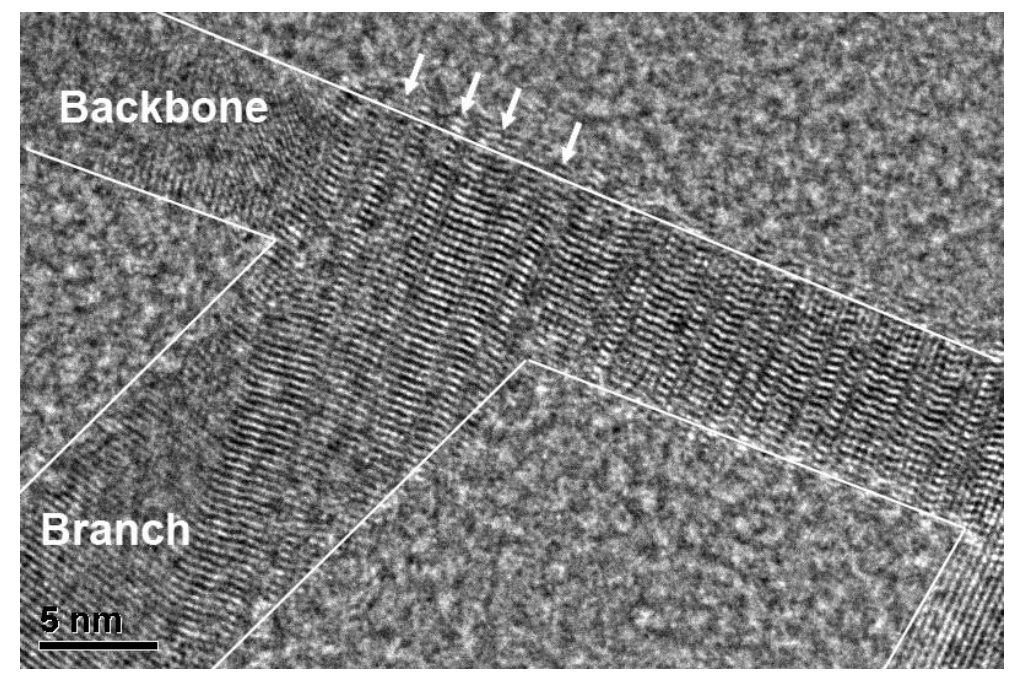

Figure S4. HRTEM image of a heterobranched CdSe-ZnSe NW with stacking faults and/or twinnings (near-vertical stripes indicated by the arrows), showing that the original stacking faults and/or twinnings in the backbone region were entirely inherited in the branch, due to the epitaxial growth process. 


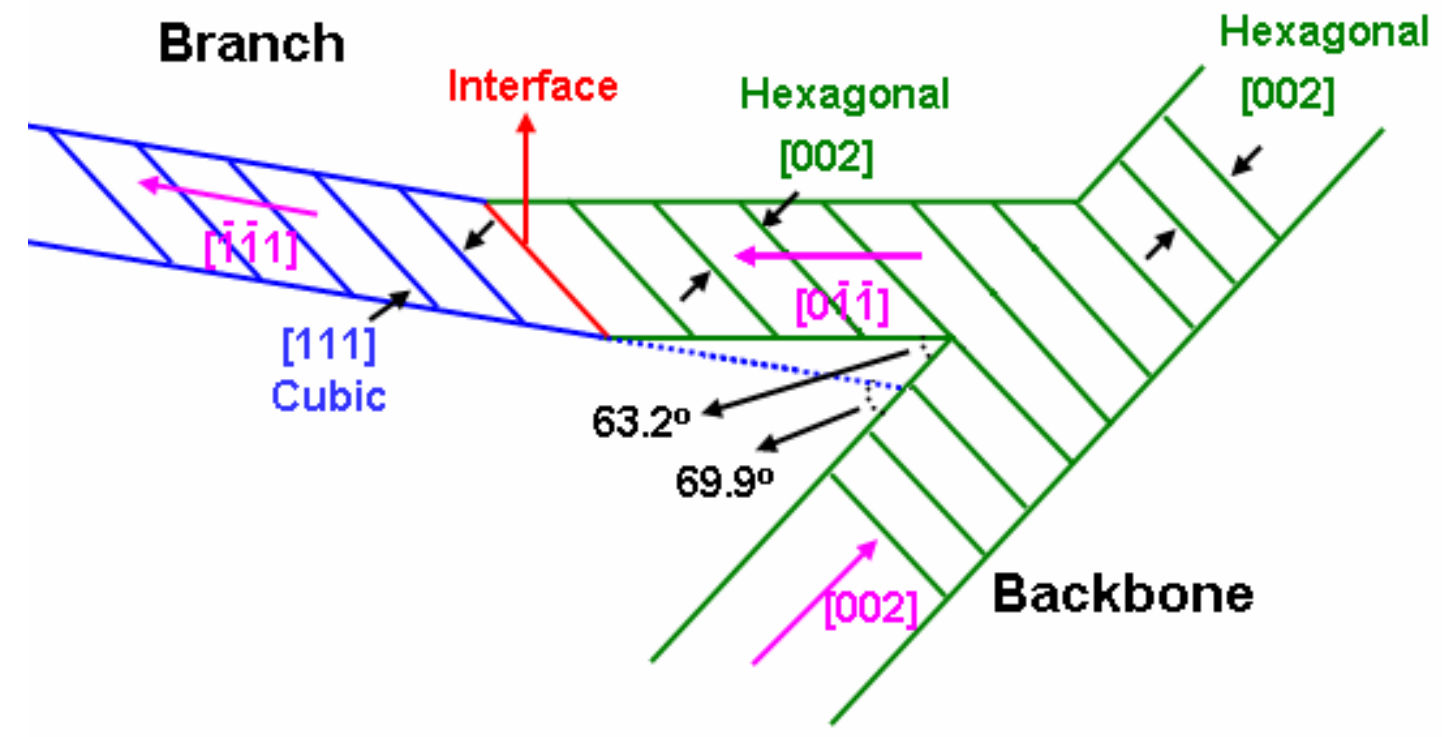

Figure S5. Cartoon illustration of the orientation and growth directions of the heterobranched CdSe-ZnSe NW shown in Figure 11. The hexagonal-structured and cubic-structured wire segments are highlighted in green and blue, respectively. The pink arrow and index indicate the growth direction for each wire segment. The red line indicates the interface between the (002) lattice planes from the hexagonal phase and the (111) lattice planes from the cubic phase. The two branch-to-backbone angles are $63.2^{\circ}$ and $69.9^{\circ}$, respectively, measured from the reciprocal lattice peaks in the corresponding FFTs (see text). 


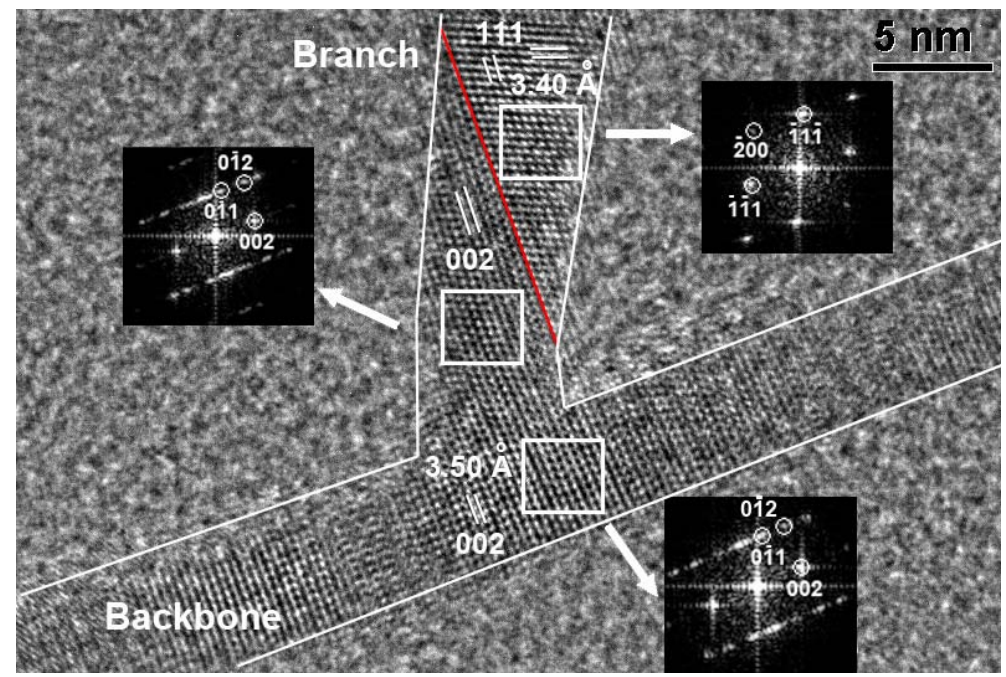

Figure S6. HRTEM image of another heterobranched CdSe-ZnSe NW in which the backbone has a hexagonal crystal structure. The red line indicates the interface between the cubic and hexagonal structures in the branch. The [002] lattice-fringe spacing of $3.50 \AA$ measured from the backbone agrees with that of CdSe, and the [111] lattice-fringe spacing of $3.40 \AA$ measured from the branch is consistent with alloyed $\mathrm{Zn}_{1-x} \mathrm{Cd}_{x} \mathrm{Se}$. The left, upper-right, and bottom-right insets show the 2D FFTs calculated from the corresponding images (indicated by squares) of the transitional branch segment, branch segment far from the junction, and backbone region, respectively. The angle between the cubic-structured branch and the hexagonal-structured backbone is $63.7^{\circ}$, determined from the reciprocal lattice peaks. This angle is about $7^{\circ}$ smaller than the predicted value $70.5^{\circ}$, indicating there is not a

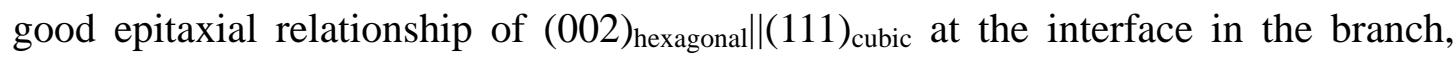
which might be caused by the slight twisting between two crystal phases at an imperfect interface. 